\title{
¿Cómo mejorar el muestreo en estudios de porte medio usando diseños con métodos mixtos? Aportes desde el campo de estudios de elites
}

\author{
How to improve sampling in medium-sized studies using \\ designs with mixed methods? Contributions from the field of \\ elite studies
}

\section{Miguel Serna}

Universidad de la República, Uruguay

miguelpsf@gmail.com (URUGUAY)

Recibido: 26.042017

Aceptado: 26.04.2019

\section{RESUMEN}

El objetivo del trabajo es abordar algunas problemáticas recurrentes y alternativas para el diseño muestral de poblaciones de porte medio en el campo de estudio de elites.

El trabajo parte de la problemática clásica de la fundamentación de los dos grandes tipos paradigmas teóricos-metodológico de muestreo, el cualitativo y el cuantitativo, revisión somera que tiene como meta mostrar la existencia de un zona de estudios de muestras de porte medio (entre 90 y 300-390 casos) donde las fronteras entre ambos se hace borrosa y por tanto es pertinente discutir la posibilidad de uso de muestro con métodos mixtos. A continuación se presenta el campo de estudio de las elites como un área fértil para el desarrollo de métodos mixtos y en particular en el procedimiento de muestreo.

Por último se describen algunos aportes desde la experiencia y práctica de investigación en el campo de las elites políticas y económicas para resolver y buscar alternativas de mejora de las muestras en poblaciones de mediano porte, a saber: a) la explicitación de criterios objetivos en la construcción empírica del objeto de estudio, b) la aplicación de juicio de expertos para la validación de la muestra, c) el uso de pares expertos como instrumento de investigación.

Palabras clave: muestreo, métodos mixtos, elites políticas, elites económicas 


\title{
PALABRAS CLAVE
}

Muestreo, métodos mixtos, elites políticas, elites económicas

\begin{abstract}
The purpose of the article is to address some recurrent and alternative problems for the sample design of medium-sized populations in the field of elite studies.

The paper starts from the classical debate of the two great types of sampling based on the qualitative and quantitative methodological-paradigms. A brief review aims to show the existence of a study area of samples of medium size (between 90 and 300-390 cases) where the boundaries between both becomes blurred and therefore it is pertinent to discuss the possibility of using samples with methods mixed The field of study of the elites is presented as a fertile area for the development of mixed methods and in particular in the sampling procedure.

Finally, some contributions from the experience and practice of research in the field of political and economic elites are described to solve and look for alternatives to improve samples in medium-sized populations, namely: a) the specification of objective criteria in the empirical construction of the object of study, b) the application of expert judgment for the validation of the sample, c) the use of expert peers as a research instrument.
\end{abstract}

\section{KEY WORDS}

Sampling - mixed methods - political elites - economic elites.

\section{INTRODUCCIÓN}

El propósito del artículo es abordar algunas problemáticas y alternativas metodológicas vinculadas al diseño muestral de poblaciones de porte medio usando métodos mixtos (cualitativos y cuantitativos) a partir de aportes y experiencias de trabajo de investigación del campo de estudio de elites.

En la primera sección se propone revisitar los principios de saturación teórica y generalización estadística, así como las diferencias más típicas en la construcción de los diseños muestrales cualitativos y cuantitativos. Más allá de la clásica dicotomía entre ambos tipos de diseños, se plantean algunas problemáticas emergentes por el desarrollo de los diseños con métodos mixtos y de desafíos empíricos abiertos ante la expansión de investigaciones que trabajan con muestras entre 90 y 300/390 casos. Se trata de estudios con muestras más grandes que los estudios cualitativos tradicionales pero no tanto como los estudios cuantitativos típicos, a lo que se suma la utilización de diseños con métodos 
mixtos que traen nuevas problemáticas para repensar la elaboración de los diseños muestrales.

En la segunda sección se describen las metodologías de investigación utilizadas desde el campo de los estudios de elites, las características típicas de su objeto, así como la pertinencia del uso de diseños con métodos mixtos. Asimismo, se plantean las alternativas metodológicas emergentes para la elaboración de los diseños muestrales en este tipo de estudios.

En la tercera sección se formulan algunos "trucos" en el oficio de investigación para la mejora y fortalecimiento del diseño muestral con métodos mixtos, a saber, a) la explicitación de los criterios objetivos para la determinación empírica del objeto de estudio, b) la utilización de controles intersubjetivos de pares expertos en el procedimiento de muestreo para la validación de la representatividad cuantitativa y saturación teórica, c) el recurso a diseños muestrales mixtos de pares expertos como instrumento de aproximación al objeto de estudio.

\section{DISEÑOS MUESTRALES CUALITATIVOS \& CUANTITATIVOS: RESPUESTAS CLÁSICAS Y ASUNTOS PENDIENTES}

En la perspectiva cualitativa más tradicional la investigación requiere un abordaje en profundidad de carácter hermenéutico, contextual y holístico para la representación de la realidad social. "Los investigadores usan los métodos cualitativos cuando creen que la mejor forma de construir una representación adecuada es través del estudio en profundidad de los fenómenos" (Ragin, 2007, pp.176). El énfasis hermenéutico se expresa en una mirada atenta a las diferencias específicas derivadas de los procesos históricos y rasgos culturales. Asimismo, se trata de una mirada holística para comparar los aspectos comunes y específicos de casos en relación a la variedad de contextos donde se desarrollan.

Para las perspectivas cualitativas más tradicionales el "examen en profundidad" y la descripción "densa" de grupos o fenómenos específicos se contraponen a los enfoques cuantitativos cuyo análisis se rige por la representación "panorámica", de corte general y estadística de la realidad social.

En la perspectiva cuantitativa clásica por el contrario, la investigación se orienta hacia el estudio de relaciones de correlación y causalidad de fenómenos sociales basados en el principio de la generalidad.

Desde la perspectiva cuantitativa, imbuidas del positivismo científico fundacional, los métodos cualitativos están restringidos a estadios exploratorios de la realidad social y de contexto de descubrimiento. Para los enfoques cuantitativos clásicos los métodos cualitativos son explicaciones insuficientes y de segundo grado de la realidad debido a la ausencia de patrones regulares y relaciones explicativas de causalidad entre variables independientes y dependientes, que no alcanzan la capacidad de verificación de hipótesis y menos de predicción general de los fenómenos sociales. 
La determinación del objeto de estudio y la elaboración del diseño muestral es un aspecto clave del debate ya clásico entre perspectivas cualitativas y cuantitativas en la investigación social.

Las decisiones referidas al momento, forma y cantidad de casos a incluir en el diseño muestral son aspecto claves del proceso de investigación cualitativa. Flick (2007) plantea como diferenciación inicial de los tipos de muestreo cualitativo, entre los que utilizan "criterios abstractos" y "criterios concretos" para la selección de casos.

Por un lado, los muestreos que se basan en "criterios abstractos" parten de una "determinación a priori de la estructura de la muestra" (Flick, 2007, pp.76). En este tipo de procedimiento de selección de casos se establecen de manera anticipada a la recogida de datos, el conjunto de celdas de tipos de grupos y cantidad de casos a relevar. Lo que se procura es reproducir un patrón de distribución de la población de estudio en forma analógica a la lógica del muestreo estadístico.

Por otro lado, los muestreos cualitativos que se basan en "criterios concretos" elaboran una "definición gradual de la estructura de la muestra" durante el proceso de investigación. El ejemplo paradigmático es el denominado muestreo teórico. En estas clases de muestras la inclusión de grupos o personas se realiza directamente vinculado al proceso de relevamiento e interpretación de los datos, y estrechamente relacionado al desarrollo de categorías analíticas y la interpretación conceptual. La cantidad de casos se determina en función de la capacidad de "saturación teórica" de ideas y categorías analíticas emergentes. En este tipo de diseños muestrales se parte del supuesto que las características y distribución de la población no se conocen de antemano y que el tamaño de la muestra no está definido previo al trabajo de campo.

Para muchos autores este procedimiento es parte sustancial de los rasgos del "proceso circular del proceso de investigación cualitativa" (Flick, 2007, pp.59). Según el autor una de las características principales del diseño cualitativo es la revisión permanente de las perspectivas sobre el objeto. Y por ese motivo, es que el autor considera a la "selección gradual como principio general en la investigación cualitativa" (Flick, 2007, pp.80), en contraste claramente con la búsqueda de la reproducción de la representación estadística de la distribución de la población típico del muestreo probabilístico.

En los procedimientos de selección de los casos se pueden encontrar en la literatura varias estrategia de muestreo deliberado (Patton, 2002), como ser la búsqueda de casos extremos o desviados, la elección de casos típicos (la mayoría o promedio); la selección de la variación máxima en el campo; la selección de casos según la intensidad de los rasgos o procesos; la búsqueda de casos críticos, políticamente importantes o sensibles; o también de la elección de los casos de más fácil acceso a la investigación.

¿Cuáles son los criterios y procedimientos utilizados para el muestreo cualitativo? ¿Qué implicancias tienen para las inferencias empíricas y análisis teóricos que se pueden desprender de estas muestras? En el trabajo con metodología cualitativas se han manejado en la literatura en general dos grandes "criterios 
maestros de muestreo" de "heterogeneidad" y "economía"(Valles, 2002, pp.68) para determinar el diseño y tamaño muestral. La finalidad del muestreo cualitativo es lograr una "representación tipológica socioestructural" en función de los objetivos del estudio.

La "heterogeneidad de la muestra" se establece de acuerdo a la selección de variables relevantes para la investigación y por procedimientos de "fijación de cuotas" para la conformación de "casilleros tipológicos". Los casilleros a completar pretenden seleccionar casos que expresen la variabilidad y diferenciación interna del campo de estudio, que pueden ser de diverso tipo.

El criterio de la economía se vincula con otros criterios como la "saturación teórica" (Glaser B.G. y Strauss A L., 1967) de cada categoría o grupos de casos estudiados, para conseguir explorar cada campo en amplitud y profundidad. El objetivo es alcanzar la reproducción de la diversidad de opiniones (en intensidad y extensión) en cada cuota, a partir de la cual adicionar mayor número de casos no agrega información sustancialmente nueva. También vinculado a la economía aparecen criterios de "naturaleza práctica" en la accesibilidad y selección de los individuos (Valles, 2002, pp.71).

El denominado "muestreo teórico" (Glaser, Strauss, 1967) prioriza como criterio el propósito y relevancia teórica de los casos que integran la muestra. El muestreo teórico no se vincula al número de casos sino que persigue la saturación de las categorías analíticas. El objetivo del mismo es desarrollar categorías emergentes y explorar sus propiedades, así como sugerir interrelaciones dentro de una teoría (Glaser, Strauss, 1967, pp.61).

A modo ilustrativo, el procedimiento de muestreo por bola de nieve, una de las estrategias más usadas para la selección de casos, no se define por la cantidad de participantes seleccionados sino por el significado y pertinencia de los datos relevados, de los participantes, los relatos y experiencias para la comprensión del fenómeno de estudio y la contribución al proceso de saturación teórica (Morse, 2007, pp.243) El investigador va identificando sujetos y sus relaciones con otros, construyendo el objeto de estudio por la redundancia o no de significados aportados en los nuevos casos que se agregan.

El muestreo se orienta a recoger la amplitud y diversidad de casos de estudio para el descubrir "diferencias fundamentales" entre grupos y obtener de esa manera la "saturación" de las categorías -que se produce no por la cantidad de casos acumulados, sino cuando casos nuevos no aportan información adicional-

En este sentido, se destaca el papel que tiene el investigador y la literatura teórica en el proceso de investigación. Más allá de diversos debates que se produjeron al interior de la propia teoría fundada, la preocupación central reside en que la construcción de categorías y conceptos debe ser un resultado emergente del propio proceso de relevamiento de los datos, más que de la influencia del propio investigador (Connor et al, 2018).

La fortaleza de esta metodología es la profundidad de la descripción de la realidad y la focalización de la mirada en pequeñas poblaciones con pocos casos pero que, o bien son bastantes homogéneas respecto algunas propiedades socia- 
les, o bien por la pertenencia a grupos sociales específicos cuando se trata de casos extremos distintos de la distribución "normal" de la población.

El muestreo teórico consiste en una construcción "progresiva de la muestra" cuyo objetivo es abordar el mundo social a través de los agentes/actores, en sus distintas "posiciones" estructurales y relacionales "jerárquicas" y "funcionales". Se trata de descubrir la "diferenciación" de roles y prácticas en "experiencias biográficas específicas" para comprender el micromundo social y descubrir los "mecanismos genéricos" en las "configuraciones específicas" de las lógicas de acción, procesos y situaciones sociales (Bertaux, 2010, pp.33).

La literatura de metodología cualitativa tiene una densa fundamentación de los procedimientos de construcción analítica del muestreo teórico y de adecuación flexible de la metodología a los objetivos teóricamente construidos por los investigadores (Wainerman, 2001, pp.43-45), sin embargo, hay mucho menos acumulación de trabajos que pongan su atención en las orientaciones prácticas que determinan el tamaño empírico efectivo de las muestra, ni tampoco que detallen acerca de cómo y cuándo se alcanza la saturación teórica,

Trabajos más recientes (Guetterman, 2015) advierten que a pesar que hay muchas recomendaciones teóricas de rigor para determinar el tamaño de las muestras cualitativas no hay muchos análisis prácticos específicos de cómo se realiza. A partir de una revisión sistemática de las muestras en estudios cualitativos realizados en las áreas de la educación y la salud el autor concluye: a) la existencia de vacíos en el detalle de los procedimientos de muestreo, b) encuentra que el tamaño promedio de las muestras se sitúa en 87 casos, aunque con diferentes prácticas de muestreo según los distintos tipos de investigación cualitativa, así como de disciplina científica; c) los estudios que incluyen la adecuación de la muestra al alcance de la saturación teórica, tienen tamaños muestrales más pequeños (53 casos promedio), aunque en niveles más altos que lo que se considera en la literatura de estudios cualitativos, d) recomendaciones de incluir: los detalles del procedimiento de muestreo y del tamaño de la muestra, sus limitaciones, las posibilidades de generalización yla adecuación a la saturación (Guetterman, 2015, pp.15-18).

En otro trabajo (Mason, 2010) también se buscó orientaciones prácticas acerca de cómo se determina el tamaño muestral en investigaciones cualitativas a través de un análisis de una base de datos de 560 estudios cualitativos (26 tipos en diversas disciplinas) para relevar el número de entrevistas realizadas durante la elaboración de tesis de doctorado. Como resultado del relevamiento empírico se estableció que el tamaño promedio de las muestras usadas en estudios cualitativos es de 31 entrevistas, superior a los mínimos de casos establecidos en la literatura de metodología cualitativa. No obstante, no existe un único patrón empírico, sino que los tamaños muestrales varían según el tipo de técnicas, por ejemplo, los promedios para la historia de vida es de 23 casos, para la teoría fundada 32 entrevistas, en la etnometodología 31 casos, y en los estudios de caso 36 entrevistas). Los tamaños muestrales más comunes los identifica entre 20 y 30 entrevistas. 
Pero más allá de los esfuerzos por determinar cuándo se alcanza la magnitud cuantitativa del tamaño muestral en estudios cualitativos, otro aspecto clave del diseño es cómo se alcanza la saturación teórica. En ese sentido, varios autores argumentan la importancia de considerar parámetros de saturación teórica que pueden hacer variar el tamaño muestral (más pequeña \& más extensa), según: a) propósito del estudio (captura temas \& desarrollo teoría), b) rasgos de la población (homogénea \& heterogénea), c) estrategia de muestreo (repetitiva \& fija), d) calidad de los datos (gruesos \& finos), e) tipo de códigos (concretos \& conceptuales), f) libro de códigos (estable \& emergente), g) meta de la saturación (identificación códigos \& significados códigos) (Hennink et al, 2016; Hennink et al, 2019)

En contraste, el diseño muestral cuantitativo tiene como meta la generalización estadística basada en la teoría de la probabilidad y el muestreo aleatorio en base a procedimientos estadísticos. El objetivo del muestreo es siempre el mismo, mejorar la eficacia y reducir el costo de una investigación mediante la selección de casos a partir de los cuáles se pueda realizar inferencias para el conjunto de la población de estudio.

La potencialidad del muestreo estadístico es que se aplica sobre poblaciones grandes, a partir de las que se selecciona en forma aleatoria muestras cuantitativas que van desde 300 o 400 casos, o más. La determinación del tamaño muestral se calcula en función de la estimación estadística de un valor cuantitativo de nivel de confianza aceptable, el establecimiento de un porcentaje de error estadístico derivado del procedimiento de muestro y la proporción estimada de algunas variables consideradas como parámetros en la población. A efectos ilustrativos, se puede mencionar algunos tamaños de muestras típicas utilizando el método de muestreo aleatorio simple, van desde un mínimo de 300 casos (con un 5,9\% de error estadístico), o 400 casos (con un 5\% de error estadístico), las muestras en tamaño estándar de 1000 casos (con 3\% de error estadístico) o las más grandes que van desde 3000 casos (con $2 \%$ de error estadístico). En la utilización de otros tipos de muestreo estadístico como los muestreos por conglomerado o estratificado en general suponen un tamaño muestral superior a 1000 casos.

La fortaleza del muestreo probabilístico se basa en la selección de una muestra de casos (n) sobre parámetros objetivos de la población para poder generalizar los resultados a todo el universo de estudio. No obstante, existen límites, como ser que las técnicas estadísticas funcionan muy bien en muestras grandes (300 o 400 según los parámetros cuantitativos mínimos generalmente aceptados, y de ahí en más) y no funciona en muestras pequeñas -60 casos o para algunos menos de 30-, más típicas de los estudios cualitativos. Tampoco se debe olvidar que más allá de las potencialidades estadísticas derivadas de la teoría de la probabilidad, no están exentos de sesgos de error no aleatorio, proveniente de la naturaleza humana, subjetiva y relacional (no cuantificable sea en la interacción encuestador-encuestado, o en la interpretación del cuestionario por parte de los encuestados, o de procedimientos que escapan a la aleatoriedad completa). 
Tabla 1: comparación rasgos típicos de diseños muestrales cualitativo y cuantitativo

\begin{tabular}{|l|l|l|}
\hline Muestreo & Cualitativo & Cuantitativo \\
\hline Objetivo & Saturación teórica & Generalización estadística \\
\hline $\begin{array}{l}\text { Determinación uni- } \\
\text { verso de estudio }\end{array}$ & $\begin{array}{l}\text { Múltiples fuentes de } \\
\text { datos, conocimiento } \\
\text { aproximativo de pobla- } \\
\text { ciones poco conocidas }\end{array}$ & $\begin{array}{l}\text { Poblaciones con caracter- } \\
\text { ísticas conocidas a través } \\
\text { de fuentes censales y es- } \\
\text { tadísticas. }\end{array}$ \\
\hline $\begin{array}{l}\text { Procedimiento de } \\
\text { muestreo }\end{array}$ & $\begin{array}{l}\text { Intencional, no alea- } \\
\text { torio }\end{array}$ & Aleatorio \\
\hline $\begin{array}{l}\text { Inferencia población } \\
\text { No probabilístico, } \\
\text { atribución de sentido y } \\
\text { significativo, } \\
\text { Alcance exploratorio }\end{array}$ & $\begin{array}{l}\text { Probabilístico, inferencia } \\
\text { estaca empírica }\end{array}$ \\
\hline $\begin{array}{l}\text { Universo de estudio y } \\
\text { tamaño de la muestra }\end{array}$ & $\begin{array}{l}\text { Poblaciones acotadas } \\
\text { de estudio pequeñas y } \\
\text { específicas } \\
\text { Muestras menores de } \\
90 \text { casos }\end{array}$ & $\begin{array}{l}\text { Poblaciones grandes } \\
\text { Muestras mayores a } \\
\text { 30on casos. }\end{array}$ \\
\hline
\end{tabular}




\begin{tabular}{|c|c|c|}
\hline $\begin{array}{l}\text { Técnicas de investig- } \\
\text { ación y número de ca- } \\
\text { sos en las muestras }\end{array}$ & $\begin{array}{l}\text { Entrevistas en profun- } \\
\text { didad } \\
\text { Promedio } 31, \text { Muestras } \\
\text { más comunes } 20-30 \\
\text { casos }(*) \\
\text { Estudios etnográficos } \\
\text { Promedio } 31 \text { entrevis- } \\
\text { tas (*) } \\
\text { Teoría fundada } \\
\text { Promedio } 32 \text { entrevis- } \\
\text { tas (*) } \\
\text { Estudios biográficos: } \\
\text { historia y relatos de } \\
\text { vida } \\
\text { Mínimo } 15 \text { (**) Pro- } \\
\text { medio } 23 \text { entrevistas } \\
\text { (*) } \\
\text { Criterios de saturación } \\
\text { teórica que pueden va- } \\
\text { riar el tamaño muestral } \\
\text { (pequeña a extensa): a) } \\
\text { propósito estudio, b) } \\
\text { población homogénea/ } \\
\text { heterogénea, c) estrate- } \\
\text { gia de muestreo repeti- } \\
\text { tiva-fija, d) calidad de } \\
\text { los datos gruesos-finos, } \\
\text { e) tipo de códigos con- } \\
\text { cretos-conceptuales, } \\
\text { f) libro de códigos } \\
\text { estable-emergente, g) } \\
\text { meta de la saturación } \\
\text { identificación códigos- } \\
\text { significados códigos } \\
\text { (***) }\end{array}$ & $\begin{array}{l}\text { Encuestas por muestreo, } \\
\text { tamaño muestra determi- } \\
\text { nado de acuerdo al nivel } \\
\text { de confianza, porcentaje de } \\
\text { error estadístico, y propor- } \\
\text { ción estimada de variables } \\
\text { consideradas como pará- } \\
\text { metros en la población. } \\
\text { Ejemplos de tamaños } \\
\text { de muestras (aleatorio } \\
\text { simple) } \\
\text { a) Nivel de confianza } 95 \% \\
\text { y } \\
5,9 \% \text { de error, } \mathrm{P}=\mathrm{Q}=50 \\
\%, \text { muestra de } 300 \text { casos } \\
\text { b) Nivel de confianza } 95 \% \\
\text { y } \\
5 \% \text { de error, } \mathrm{P}=\mathrm{Q}=50 \\
\% \text {, muestra de } 400 \text { casos } \\
\text { c) Nivel de confianza } 95 \% \\
\text { y } \\
3 \% \text { de error, } \mathrm{P}=\mathrm{Q}=50 \\
\%, \text { muestra de } 1000 \text { casos } \\
\text { d) Nivel de confianza } 97 \% \\
\text { y } \\
2 \% \text { de error, } \mathrm{P}=\mathrm{Q}=50 \\
\%, \text { muestra de } 3000 \text { casos } \\
\text { Tipos de muestreo por } \\
\text { conglomerados y estrati- } \\
\text { ficados en general tamaño } \\
\text { muestras superior a } 1000 \\
\text { casos. }\end{array}$ \\
\hline
\end{tabular}

Nota al pie: (*) Mason (2010) (**) Bertaux (2010) (**) Hennink et al (2016)

La breve revisión previa tuvo como sentido identificar las diferencias principales teóricas (saturación teórica \& generalización estadística) y metodológicas (tipos de población, procedimientos de selección (deliberado \& aleatorio) y muestreo (no probabilístico \& probabilístico) entre las muestras cualitativas y cuantitativas. Las diferencias se expresan también en tamaños muestrales 
dispares, entre muestras cualitativas pequeñas (menores a 30 o 60 casos), y las muestras cuantitativas estadísticas (mayores a 300 o 400 casos).

Pero una mirada atenta del tamaño muestral descubre que existe una zona borrosa en el medio, de dudosa clasificación, un conjunto muy amplio de estudios que trabajan en 90 y 300 o 400 casos. Más allá de la oposición tradicional entre las perspectivas cualitativa y cuantitativa "ortodoxas", los avances en el procesamiento informático para la codificación de categorías analíticas y la posibilidad de aplicación de múltiples técnicas de análisis para la comprobación de hipótesis son promisorias por su potencialidad de análisis cuantitativos de datos cualitativos(), así como la expansión de estudios cuantitativos no típicamente aleatorios, con fuentes de datos y tecnologías que tienen criterios cualitativos en la selección de casos, abre nuevos campos para el uso de diseños muestrales con métodos mixtos.

La expansion de prácticas de integración y triangulación de métodos cualitativos y cuantitativos se acompañó del desarrollo de la fundamentación teórica y metodológica de los métodos mixtos (Creswell, 2009, Fetters, Molina-Azorin, 2017). La estrategias de utilización de los métodos mixtos pueden ser tanto por la triangulación simultánea como por el uso secuencial de metodologías cualitativas-cuantitativas o cuantitivas-cualitativas (Creswell, 2009, pp.207-210)

La utilización de métodos mixtos involucra a todo el diseño y el proceso de investigación, desde la validación de las metodologías, la recolección de datos hasta el análisis e interpretación. En ese sentido, es pertinente la discusión de la posibilidad de uso de métodos mixtos en el diseño muestral, y que ello es objeto de creciente atención en la literatura especializada (Sykes et al, 2018, pp.8). Lo anterior no quiere decir que los métodos mixtos estén exentos de críticas, ni que sea sencillo la articulación de metodologías cualitativas y cuantitativas (Verd, López, 2008).

\section{DISEÑOS CON MÉTODOS MIXTOS: NUEVOS DESAFÍOS PARA EL MUESTREO DESDE EL CAMPO DE LOS ESTUDIOS DE ELITES}

El estudio de elites políticas y económicas ofrece algunos desafíos comunes a la hora de su abordaje con métodos de investigación social. Se tratan de dirigentes que tienen una alta visibilidad pública y status social, pero al mismo tiempo las arenas y tramas de poder son opacas en su funcionamiento cotidiano.

La construcción del campo empírico de la población de estudio es un trabajo arduo, complejo y denso sobre las fuentes de datos diversas. Las dificultades en la identificación y acceso al trabajo de campo de las elites es tan sólo el primer paso para determinar con qué tipo de dirigentes y cuál elite se va a trabajar, el siguiente casi tan importante y estrechamente vinculado al marco muestral o lista de casos que integran el universo de estudio es la elección del diseño muestral. 
En este tipo de estudios en general no hay censos o marcos muestrales oficiales, ni universos poblacionales grandes que permitan utilizar las clásicas muestras de corte probabilístico. De hecho, en muchas investigaciones que trabajan sobre universos de estudio pequeños la población objetivo no es completamente conocida, ni tampoco se conoce en profundidad y en forma estandarizada las características de la población. Por tanto, el tamaño de la muestra no está determinado con antelación y los procedimientos de muestreo son dirigidos de acuerdo a los objetivos de la investigación y deben ser definidos paso a paso. Por otra parte, los desarrollos más recientes trabajan con universos de estudio y muestras cuantitativas expandidas de porte medio, aunque no necesariamente bajo el formato de las muestras probabilísticas típicas. Es por tanto, un campo fértil para la utilización de métodos mixtos, combinando las metas de saturación teórica de los diseños cualitativos con las pretensiones de generalización estadística de los diseños cuantitativos.

Para poder identificar el campo de estudio es útil y necesario comenzar precisando los criterios teóricos para la reconstrucción empírica de las elites, algunos son particularmente útiles para la elaboración de las listas de dirigentes. Está bastante aceptado en la literatura especializada sobre elites (Hoffmann, 2018, Codato 2016) la utilización de tres métodos principales para la identificación de la elites a) la posición institucional, b) la reputación social y c) la capacidad efectiva en toma de decisiones.

El primer método, es la utilización del criterio altimétrico, de la posición jerárquica de mando que define un grupo de personas que ocupan posiciones de autoridad en la cúspide de instituciones y estructuras de poder centrales en el orden social. Casi todas las teorías de las elites, independientemente de las diferencias de interpretación, parten para la definición operativa de las elites del criterio posicional jerárquico de mando. La utilización del término altimétrico da cuenta no sólo de las posiciones más altas en la cúspide del orden social, sino también de las variaciones relativas de distancia entre dirigentes y dirigidos en la "topografía" de instituciones y estructuras de una sociedad.

No obstante, los otros dos métodos se presentan porque la jerarquía formal en las instituciones no es la única fuente de poder. Hay personas cuya "reputación o prestigio social" le dan liderazgo y poder de influencia más allá de la ocupación de los puestos de autoridad, este es el objetivo del método reputacional como criterio para la identificación de dirigentes. El tercer método para identificar elites consiste en el análisis de los procesos de toma de decisión, que no siempre se restringen o explicitan en las normas, estructuras y competencias formales, sino también abarca las dinámicas de poder de círculos de trato personal, relaciones íntimas y de confianza producido en pequeños círculos y grupos sociales de dirigentes fuera de los espacios públicos e institucionalizados.

Una vez explicitados los métodos teóricos para la identificación de elites es preciso avanzar en su operacionalización empírica. Si por un lado el método posicional aparece como más preciso y objetivo, por el otro lado, los métodos de la reputación y la decisión depende de la determinación de las redes visibles e invisibles del poder objeto de la investigación. El asunto es cómo articular los 
diversos criterios para la investigación sin caer en el simple objetivismo de la autoridad formal ni en el subjetivismo de las pretensiones de reputación de los sectores altos o las arenas ocultas e íntimas del ejercicio del poder. Así pues, desde el comienzo en la determinación del objeto estudio, este campo de investigación trabaja con criterios objetivos y subjetivos, de articulación de métodos cuantitativos y cualitativos.

A primera vista definir los grupos dirigentes, "quienes mandan", es una tarea simple, son los que ocupan posiciones en la cúpula de la jerarquía social, del poder político, del poder económico, etc. No obstante, dichas posiciones no son tan cristalizadas como pueden aparecer en las estructuras institucionales, con relaciones intersticiales entre grupos dirigentes con los recursos y diversos campos de poder.

En función de ello, en esta etapa inicial una recomendación bastante fundamentada en la literatura sobre metodologías cualitativas (Thomas, 1995, pp.12) es la búsqueda de distintas fuentes de información y la mayor cantidad de datos posibles sobre las elites. En ese sentido varias son las técnicas más comunes en este campo de estudios.

El estudio directo de los actores con metodología cualitativas, particularmente con la entrevistas en profundidad "especializadas a elites" (Dexter, 1970) es recomendado para que promueva que el entrevistado sea el protagonista de la investigación señalando su perspectiva sobre el problema y la situación. Ciertamente, el tema del acceso directo a los entrevistados en este campo coloca un problema adicional en la relación entrevistador y entrevistado debido a que las elites tienden a tratar de tener un control de la entrevista, el tiempo de duración y el modo de responder, con mucho mayor énfasis que otros grupos sociales (Harvey, 2011, pp.439). En ese sentido se han realizado advertencia como la necesidad de protección de la integridad de la investigación y el investigador (Ostrander, 1995, pp.148). Más allá de las precauciones y advertencias para su uso, lo que sí está claro que la técnica cualitativa de entrevistas en profundidad es pertinente y de uso generalizado por su potencialidades para el estudio de las trayectorias, saberes y prácticas de las elites (Gené, 2014)

Otra técnica tradicional en los estudios de elite proviene del campo de la historia denominado como método prosopográfico (Lawrence Stone, 2011) o biografía colectiva, cuyo objetivo es estudiar los perfiles sociales y características comunes de un grupo social, una categoría profesional o colectividad a través del estudio de las genealogías históricas, trayectorias biográficas y cursos de vida de sus miembros. El método consiste en el estudio de variables empíricas sobre el origen social que contribuya a entender los rasgos comunes del grupo; su relación con la dinámica de las instituciones del orden social en un tiempo histórico y espacial determinado.

La biografía colectiva como método de las ciencias sociales, también tiene su tradición en los estudios de elites. En ese sentido se quiere colocar el acento en la perspectiva sociológica del método biográfico, que significa encontrar las “coincidencias" sociales en las biografías (Becker, 2009, pp.49), las pautas recurrentes de la experiencia y las relaciones sociales de la vida cotidiana más allá 
de lo específico de cada biografía individual. De esta forma, se busca desplazar la perspectiva cognitiva de los debates teóricos clásicos de la sociología que centraron la contraposición subjetivismo versus objetivismo, individualismo versus holismo, hacia el énfasis de la mirada en las relaciones sociales, en donde se intersecta lo individual y lo colectivo (Corcuf 2013, pp.27-28 y 131); el análisis de las posiciones estructurales y el de las prácticas de los individuos y grupos.

Desde la sociología de las elites se desarrollaron en los últimos tiempos análisis sociográficos de las propiedades sociales de origen (social, trayectoria profesional, etc.) de las elites políticas y económicas para poder identificar perfiles sociales y comprender aspectos tales como el modo de reclutamiento de los miembros (más abierto o cerrado), el grado de cohesión interna como grupos sociales (formación educativa, socialización e integración sociocultural) y las relaciones interelites (Hartman, 2007, Genyes, 2011).

En las últimas décadas varios estudios de elites abordan el reclutamiento, la composición y las trayectorias biográficas de las elites políticas y económicas, utilizando para ello los métodos de la biografía colectiva. A modo de ejemplo, existe una acumulación importante de estudios de elites en Brasil (Codato, Perissinotto, 2016); o se puede citar desde la ciencia política estudios focalizados en el reclutamiento y las carreras políticas de las elites (Alcántara, Barragán, Sánchez, 2018). En estos tipos de estudio articulan análisis cualitativo de tipos de trayectorias con análisis cuantitativos de patrones regulares de carreras políticas.

$\mathrm{El}$ análisis de redes es otra técnica de creciente uso para el abordaje de elites políticas y económicas, en especial para identificar posiciones, relaciones y redes intra e interelites (Keller (2018), Cárdenas, (2014)). También en este caso el desarrollo informático ha estimulado el uso combinado de análisis de cartografías espaciales con análisis cuantitativos de indicadores de proximidad y distancia entre agentes y nodos.

En el estudio de elites también está presente el uso de encuestas de opinión como técnica cuantitativa aplicada a universos y poblaciones específicas de dirigentes, como grupos particularmente relevantes en ámbitos decisorios. Se pueden encontrar varias iniciativas relevantes de alcance comparado a nivel regional de varios países y también a nivel nacional, que comienza a establecer una acumulación de bases de datos de encuestas orientadas específicamente al estudio de las elites (Rodríguez-Teruel, Daloz (2018)). Además aparecen estudios que comparan la opinión de las elites con las de los ciudadanos, para analizar distancias y proximidades entre ambos grupos de opinión.

Esta somera revisión de técnicas más frecuentemente usadas en el campo de estudio de élites tuvo como sentido mostrar que es un área de investigación particularmente fértil para la articulación e integración de metodologías cualitativas y cuantitativas. Asimismo, y a los efectos del presente artículo de mostrar que es un campo que trabaja con poblaciones y muestras de porte medio, donde se presentan muchas veces en la formulación del diseño muestral, en forma superpuesta los objetivos de saturación teórica y los de generalización estadística. En este sentido, se propone para la siguiente sección recorrer algunos mecanismos usados para fortalecer el diseño muestral en este tipo de estudios. Así pues, se 
busca abordar la problemática explorando los "trucos" del oficio (Becker, 2009), entendiendo por tales las operaciones y procedimientos que se utilizan para sortear y resolver las dificultades de problemas comunes y recurrentes en la metodología de investigación cualitativa a partir de aportes provenientes del campo de estudio de las elites.

Tabla 2: rasgos emergentes diseño muestral con métodos mixtos desde el campo de estudio de elites

\begin{tabular}{|l|l|}
\hline Objetivo & Diseño muestral con métodos mixtos \\
\hline $\begin{array}{l}\text { Saturación teórica y Generalización cuan- } \\
\text { titativa }\end{array}$ \\
\hline tudio & $\begin{array}{l}\text { Múltiples fuentes de datos, conocimiento } \\
\text { aproximativo de poblaciones poco cono- } \\
\text { cidas } \\
\text { Tres métodos: posicional, decisional y } \\
\text { reputacional }\end{array}$ \\
\hline Procedimiento de muestreo & $\begin{array}{l}\text { Criterios objetivos, intersubjetivo, cono- } \\
\text { cimiento experto }\end{array}$ \\
\hline Inferencia población & $\begin{array}{l}\text { No probabilístico, atribución de sentido y } \\
\text { significativo, } \\
\text { Alcance cualitativo y cuantitativo }\end{array}$ \\
\hline Tamaño universo y muestra & $\begin{array}{l}\text { Universos determinados con poblaciones } \\
\text { de estudio específicas } \\
\text { Muestras entre } 90 \text { y 400 casos }\end{array}$ \\
\hline $\begin{array}{l}\text { Técnicas de investigación más } \\
\text { usadas en campo estudios de } \\
\text { elites }\end{array}$ & $\begin{array}{l}\text { Entrevistas en profundidad } \\
\text { Prosopografía histórica } \\
\text { Biografía colectiva y trayectorias sociales } \\
\text { cualitativo-cuantitativo } \\
\text { Análisis de redes cualitativo-cuantitativo } \\
\text { Carreras políticas, análisis cualitativo- } \\
\text { cuantitativo } \\
\text { Encuestas de opinión }\end{array}$ \\
\hline
\end{tabular}




\section{TRUCOS PARA LA MEJORA DEL MUESTREO DE ELITES POLÍTICAS Y ECONÓMICAS CON DISEÑOS CON MÉTODOS MIXTOS}

En esta sección se van a presentar tres trucos utilizados en estudios específicos de elites políticas y económicas para mejorar el diseño muestral en poblaciones específicas de porte medio utilizando métodos mixtos.

\subsection{La búsqueda de la objetividad en los registros biográficos y la explicitación de criterios teóricos cualitativos en la construcción del universo de estudio.}

La construcción del registro biográfico de dirigentes es en cierta medida la primera elección de investigación, en el sentido que son grupos sociales relativamente pequeños, y plausibles de identificación personal. Y al mismo tiempo porque son grupos de los que generalmente no hay estadísticas e información cuantitativa sistematizada como sucede en el conjunto de la población, y al mismo tiempo se trabaja con múltiples fuentes de datos.

Como se señaló previamente cualquier definición operacional de las elites parte del método posicional utiliza el criterio objetivo jerárquico altimétrico de personas que ocupan posiciones en la cúspide de poder de la sociedad. Para la definición de las posiciones jerárquicas en instituciones de poder se requiere además tener en cuenta la temporalidad y persistencia en el tiempo de la ocupación y desempeño de posiciones sociales de poder.

Estos aspectos generalmente se establecen al momento de la conformación de la lista de dirigentes a estudio. A nivel de las elites políticas se suele proceder a la identificación de personas a partir de la lista de posiciones puestos jerárquicos en instituciones políticas, como por ejemplo, el parlamento, el gobierno o los partidos políticos. Esta decisión puede ser simple para el estudio comparado, tanto en trabajos previos de varios países (autor/a et al, 2007, autor/a et al 2012) como en proyectos comparados como el observatorio de elites parlamentarias latinoamericanas del Instituto de Iberoamérica de la Universidad de Salamanca que abarca parlamentos de 18 países en un período desde 1993 a la actualidad(), en cualquiera de los estudios el número de casos es suficiente para el análisis cualitativo y cuantitativo.

Pero si se concentra en un solo país la pequeñez cuantitativa de los grupos de elite aparece en forma inmediata. Por ejemplo, en un país pequeño como Uruguay ambas cámaras parlamentarias tienen 130 miembros, más un elenco de gabinete ministerial que suma alrededor de 30 integrantes más. Por tanto, para llegar a un número cuantitativo significativo (que supere las poblaciones pequeñas) se deben considerar todos los órganos de representación política y/o incorporar en la dimensión temporal tomando períodos más largos de dos, tres o más 
mandatos de gobierno (Autor/a et al, 2012). A modo ilustrativo, para el período 2000-2015 la base de políticos construido alcanzó a 376 personas.

Paso seguido se realiza una revisión cualitativa de múltiples fuentes de datos. Para comenzar de las instituciones en las que los dirigentes ostentan posiciones de autoridad. Las instituciones cuentan con documentos variados, desde archivos, anuarios y memorias hasta los portales web. La información disponible proporcionada por las propias organizaciones no es siempre suficiente ni pertinente para la investigación, por dichos motivos y otros, es útil recurrir a fuentes de datos personales. Este tipo de información es muy variada, desde documentos y publicaciones personales, curriculum vitae, entrevistas en medios de comunicación, hasta la información secundaria disponible en internet y redes virtuales. Información con una impronta subjetiva no estandarizada, pero que es una rica fuente datos para reconstruir el origen y la trayectoria biográfica de los dirigentes.

A medida que avanza la investigación sobre los elencos dirigentes se transita desde un enfoque estructural de posiciones objetivas de autoridad hacia un abordaje cualitativo centrado en comprender el entramado de relaciones sociales de los grupos dirigentes.

La revisión de las fuentes documentales es una tarea ardua de búsqueda de información biográfica, generalmente no sistematizada ni estandarizada, donde se hace necesario seleccionar lo relevante de lo accesorio respecto a las variables de estudio; así como la distinción entre la autopresentación personal del individuo y el reconocimiento institucional del estatus y liderazgo atribuido; el relato de vida; entre otros aspectos.

La creciente utilización de internet brinda un repositorio y repertorio diverso de documentos biográficos disponibles. A modo ilustrativo para los políticos en general una fuente de información se encuentra en los portales oficiales de los Parlamentos. En los mismos es frecuente encontrar datos biográficos de corte personal, y de actuación o desempeño parlamentario de los políticos. En cuanto a los datos biográficos suelen aparecer indicadores sociodemográficos (lugar de nacimiento, sexo, edad, color de piel en la foto, etc), de formación educativa, así como información referida a la trayectoria y carrera personal en puestos de reconocimiento público (cargos en el Estado, y posiciones de destaque en el ámbito político y también en la sociedad, especialmente en la sociedad civil organizada -gremios, asociaciones, etc.-). Si bien es frecuente encontrar estos datos, vale la pena recordar que no se encuentran en forma estandarizada, ni en igual profundidad y detalle para todos los dirigentes, en muchos casos ni siquiera están disponibles.

En el repertorio de datos biográficos la recopilación del curriculum vitae merece particular atención. Por la forma de construcción procura reflejar desde la perspectiva subjetiva de cada dirigente su formación y trayectoria profesional en la vida pública. Nuevamente se advierte que no se trata de documentos estandarizados ni en forma ni en extensión.

Luego entre las posiciones de autoridad política están los cargos de gobierno y los cargos partidarios. A pesar de que los puestos ejecutivos tienen una mayor 
relevancia por su poder y autoridad, los portales oficiales generalmente cuentan con menor información sobre la biografía de los gobernantes y más sobre los actos de gobierno. Por otro lado, se encuentran las páginas web de los partidos que son mucho más heterogéneas en cuanto a la información disponible y la forma de comunicación. Existen páginas muy completas que ponen a disposición on line todos aspectos de la vida partidaria, desde la información de posiciones y programas políticos, pasando por los órganos ejecutivos y estructuras organizativas, las resoluciones y asambleas o congresos, hasta el detalle de los dirigentes.

Otro ejemplo contrastante, es el análisis de los empresarios, en estos casos los portales oficiales de las empresas son más impersonales, donde suele haber información sobre la empresa, su organización, posicionamiento económico en el mercado y comunicación pública con usuarios y proveedores, pero muy escasa o nula sobre sus cuadros dirigentes y sus perfiles biográficos.

Otra fuente de datos proviene del papel de los medios de comunicación en la construcción de biografías de los personajes públicos, por ejemplo a través de entrevistas y notas de prensa. Recursos on line que son cambiantes en el tiempo, de acuerdo a la dinámica propia que adquieren las tecnologías de información y comunicación.

La presencia de dirigentes en los medios de comunicación masiva, así como la existencia de libros sobre la semblanza biográfica de los mismos es en sí mismo un indicador de diferencias jerárquicas de liderazgo dentro de grupos dirigentes. A modo ilustrativo, para los políticos el uso del discurso y sus componentes performativos constituyen un elemento central para conformación de los líderes y un insumo para el análisis del discurso de los mismos (autor/a et al, 1995).

En otro ejemplo, para el caso el estudio de elites económicas (Autor/a et al, 2015) tuvo como objetivo realizar una cartografía social de las trayectorias de directivos y altos ejecutivos de las principales empresas de los sectores más dinámicos del Uruguay en los últimos 10 años. Luego se relevó información básica de las mismas y del sector productivo de actividad, al tiempo que se buscó trazar un mapa de las relaciones entre ellas. No obstante, pasar de la información económica-contable a la organización humana de las empresas no fue tarea simple.

Para estos fines la búsqueda en internet fue una herramienta fundamental de trabajo y las páginas web de las empresas la fuente de información inicial. A partir de allí se relevó la presentación pública de las empresas, su visión y misión, el organigrama, responsabilidad social empresaria, entre otros. A pesar de ello, la identificación e información acerca de las personas que integraban los puestos de mayor jerarquía, no estuvo disponible en la gran mayoría de los casos. Contrariamente a lo que se sostiene en la literatura respecto a que las corporaciones trasnacionales tienden a hacer público el listado de sus altos ejecutivos (Useem, 1995, pp.18) en el caso de Uruguay no se verificó, siendo excepcionales los casos que eso sucedió. De allí que fuera necesario buscar otra serie de estrategias alternativas para sortear las trabas impuestas por lo que se debió recurrir a búsquedas más profundas y diversificar las fuentes de datos. 
Así pues, se recurrió a una herramienta clásica como son los obituarios, para observar quienes envían condolencias al fallecido cuando se nombran a los directores de las empresas, presidentes, vicepresidentes y altos gerentes. Los obituarios resultan muy útiles para aportar información acerca de las relaciones entre las empresas y familias, las relaciones sociales y estatus de los distintos miembros.

Asimismo, la búsqueda en redes sociales virtuales como LinkedIn permitió identificar a gerentes generales y de división, en tanto se trata de una red social donde la prioridad la tiene el ámbito laboral y profesional, por lo que las personas se identifican en función de los puestos que ocupan en las empresas -ventaja que posiciona a esta red social por encima de Twitter y Facebook para los propósitos de nuestro estudio-. LinkedIn fue de utilidad además para el relevamiento del curriculum vitae de los altos ejecutivos de las empresas, aun cuando en la mayoría de los casos solo se pueda acceder a versiones abreviadas de los mismos.

La construcción del registro biográfico debe pues considerar múltiples dimensiones, desde aspectos como motivaciones, hábitos de comportamientos y estilos de vida, actitudes, representaciones, creencias e ideologías, el origen social, la formación educativa y las trayectorias (ocupacionales, educativas, carreras en posiciones de autoridad y poder). El objetivo de la pasar de la biografía individual a la biografía colectiva, de lograr descubrir los tipos sociales e identificar la trama intersubjetiva de patrones comunes de posiciones y relaciones sociales, así como los mecanismos de socialización y formación de grupos y actores en situaciones sociales y contextos específicos.

La conformación de bases y matrices de datos con información biográfica cualitativa permiten sistematizar una serie de dimensiones relevantes para el estudio de las élites políticas y económicas, y es la base empírica para la aplicación de técnicas de análisis cualitativo y cuantitativo. En este sentido, es imprescindible para la construcción de los datos cualitativos fiables pasa por una cuidadosa revisión a partir de las diversas fuentes de datos acuerdo a las dimensiones y criterios teóricamente relevantes.

\subsection{LOS CONTROLES INTERSUBJETIVOS DE EXPERTOS PARA LA VALIDACIÓN DEL DISEÑO MUESTRAL CON MÉTODOS MIXTOS}

El siguiente mecanismo para fortalecer el diseño muestral con método mixtos consiste en la incorporación de los criterios cualitativos de corte intersubjetivo, para validar el procedimiento y tamaño de la muestra. Se habla de criterios intersubjetivos porque suponen la incorporación de otras perspectivas cualitativas de pares expertos que se cruzan entre sí y que son ajenas e independientes de los eventuales sesgos subjetivos del investigador. Es aquí donde se encuentra la importancia cualitativa de los informantes calificados y el papel del 
test de jueces como herramientas para fortalecer la representatividad cuantitativa y cualitativa de la muestra.

A modo ilustrativo, en un estudio sobre dirigentes políticos de partidos de izquierda en Argentina, Brasil y Uruguay (1983-2002) a partir de un listado de dirigentes partidarios, candidatos a cargos ejecutivos nacionales y parlamentarios electos (que sumaban algunos cientos de personas) se utilizó un test de jueces con académicos especializados en la temática e informantes calificados, lo que permitió seleccionar una muestra de políticos con mayor liderazgo y autoridad dentro de las organizaciones políticas e incorporar también algunos casos de políticos que tenían un liderazgo y reputación personal más allá de ocupar o no puestos de autoridad política (Autor/a, 2004). Siguiendo este procedimiento se pudo trabajar con una muestra razonable del punto de vista cualitativo y cuantitativo, de 206 dirigentes políticos, 39 de Argentina, 103 de Brasil, y 64 de Uruguay. Las submuestras recogían así tanto las diferencias de representación política de partidos de izquierda entre los tres países, así como de los legados y trayectorias históricas de cada país.

Otro ejemplo, en un estudio sobre elites económicas en Uruguay (Autor/aet al, 2015), la selección de 50 empresas más relevantes en una década incorporó también el test de jueces con informantes calificados que permitió balancear los dos criterios objetivos de las estadísticas oficiales referidos al tamaño de los emprendimientos como ingreso anual y número de ocupados con otros datos cualitativos significativos para determinar la relevancia económica de una empresa, como ser los sectores económicos y posiciones estratégicas dentro del mercado, la existencia de inversión extranjera directa, la estructura propietaria y grupos económicos dueños de las empresas, así como para descartar empresas no relevantes.

La utilización del test de jueces expertos e informantes calificados no sólo es útil para la selección con criterios intersubjetivos de personas para las muestras, sino también que ellos mismos van agregando datos cualitativos y redes de contacto para acceder a los mismos con técnica de entrevistas personales. En estos casos, la confección de una submuestra para las entrevistas en profundidad obedece más claramente a lo lógica de muestreo cualitativo, como ser la selección de casos típicos en términos de diversidad y heterogeneidad de patrones existentes dentro del campo de estudio de elencos dirigentes.

En definitiva, este mecanismo (y otros análogos que pueden desarrollarse) permiten mejorar la consistencia interna marco y fortaleza diseño muestral cualitativo, incorporando criterios intersubjetivos mediante juicios, que son al mismo tiempo un mecanismo de control de los sesgos proveniente de la subjetividad del investigador. De hecho se puede mencionar que este tipo el juicio o panel de expertos ya se aplica en investigaciones para la validación de contenido en instrumentos de medición por ejemplo de indicadores para un test cuantitativo de actitudes psicológicas (Escobar-Perez, Cuervo-Martinez (2008)). La novedad propuesta en las prácticas referidas es su extrapolación del mecanismo de juicio de expertos en el proceso de selección y elaboración del tamaño muestral con métodos mixtos. 


\subsection{La muestra cuantitativa-cualitativa de expertos para la investigación de elites.}

La opinión de comités de expertos ya son utilizado como técnica de investigación cualitativa (por ejemplo el panel de expertos según la metodología cualitativas DELPHI), no obstante pueden ser potencialmente útiles en distintas metodologías, y en particular para el desarrollo de diseños muestrales con métodos de investigación mixtos.

La elección de especialistas para el estudio de elites es una de las metodologías que se usa para identificar y medir los grados de influencia de grupos de minoritarios de poder en contextos históricos sociales determinados. Es el caso del método de la reputación que tiene como objetivo la identificación de elites según del grado de influencia y reconocimiento de estatus social que tienen en una sociedad determinada.

En cuanto al diseño muestral mixto se puede ilustrar un estudio comparado sobre ocho países de América Latina realizado en el segundo semestre de 2017 (autor, et al, 2019), se aplicó una encuesta "on line" por email a expertos en ciencias sociales (economistas, politólogos y sociólogos) para identificar las estrategias y prácticas más frecuentes de las elites empresariales de salvaguarda de sus intereses y captura de recursos económicos en las políticas públicas y las consecuencias sobre democracia. La elección de expertos se realizó por su conocimiento especializado y cercano del objeto de estudio.

En cuanto al diseño muestral tiene varios aspectos para clasificarla como una muestra con métodos mixtos. Para la construcción del universo de expertos se utilizaron criterios objetivos a saber, se partió de una selección de 63 universidades y centros de investigación público y privados con desarrollo relevante en ciencias sociales, se consideró la antigüedad de los mismos, la diversidad institucional y de regiones existentes en cada uno de los ocho países de acuerdo a un estudio comparado de instituciones de educación superior realizado por CRESALC-UNESCO. En cada una de las universidades seleccionadas se buscaron los 119 programas de investigación y de posgrado existen en las tres disciplinas elegidas (economía, ciencia política y sociología) y a partir de ahí se construyó una lista de 2500 investigadores expertos priorizando a los permanentes, con formación de doctorado e investigación en las temáticas de estudio. Paso siguiente, se estableció previamente cuotas mínimas por país variables según tamaño y peso relativo de los ocho países elegidos

La etapa posterior consistió en invitar a responder un cuestionario con 25 preguntas (4 de respuesta abierta) mediante un correo electrónico personalizado a los integrantes de la base de datos a responder la encuesta en forma on line. Como resultado se obtuvo una tasa de respuesta del 11,5\% con una muestra de 268 personas. Así pues, se trató de una muestra con métodos mixtos: una muestra cuantitativa no aleatoria, de porte medio por debajo del límite del tamaño estándar de las muestras estadísticas clásicas e incorporó un conjunto de criterios cualitativos y objetivos en su construcción. 
Por otro lado, los resultados de la investigación de la percepción y opinión de los expertos en la temática fueron muy auspiciosos tanto por la receptividad a la técnica como en términos de resultados empíricos identificación actores relevantes, tipo de influencia y prácticas más frecuentes, así como consecuencias para las políticas públicas. En lo que refiere al diseño muestral es de destacar la combinación en la muestra de los principios de saturación teórica en la utilización de criterios teóricos y fijación de cuotas y de la generalización cuantitativa no aleatoria para mejora la representatividad de la muestra.

\section{CONCLUSIONES}

El propósito del trabajo consistió en abordar problemáticas recurrentes y alternativas para el diseño muestral de poblaciones de porte medio en el campo de estudio de elites.

Al principio se planteó una comparación entre los dos grandes paradigmas teóricos-metodológicos para el muestreo, el cualitativo centrado en el principio de saturación teórica y el cuantitativo cuyo eje es el principio de generalización estadística. Ambos se operacionalizan en universos de estudios diferentes, entre poblaciones pequeñas y homogéneas y poblaciones grandes y heterogéneas; y luego en tamaños muestrales bien distintos, entre muestras cualitativa y cuantitativamente pequeñas y muestras cuantitativas y estadísticamente grandes. Más allá de las diferencias teóricas y de alcance empírico, se planteó una zona intermedia donde las diferencias entre los criterios cualitativos y cuantitativos son borrosas, se entremezclan y combinan en forma creciente. Justamente es en esta zona donde han prosperado los estudios de elites y desde donde se presentan diseños con métodos mixtos.

El campo de estudios de elites debido a los rasgos de su objeto estudio se caracterizó por la combinación de métodos múltiples para la identificación de dirigentes (posicional, reputacional y decisional), así como por la utilización de metodologías cualitativas de investigación social, como las entrevistas en profundidad, la proposografía histórica y diversas variantes de la biografía colectiva desde la sociología, y la ciencia política. Pero también por el uso de metodologías cuantitativas de análisis de datos cualitativos, de metodologías cualitativas y cuantitativas de análisis de redes, así como de las encuestas de opinión cuantitativas. Es decir, ha sido un campo fructífero para el ensayo de diseños de investigación con métodos mixtos. En particular, se focalizó la problemática del muestreo y las alternativas metodológicas emergentes para la elaboración de los diseños muestrales en poblaciones de porte medio.

Como cierre, se presentan alternativas emergentes recogidas de la experiencia práctica de algunos estudios concretos con el objetivo de sistematizar "trucos" en el oficio de investigación para la mejora y fortalecimiento del muestreo con métodos mixtos.

En primer término, la pertinencia de utilizar criterios objetivos en la construcción de la lista de los registros de casos como marco muestral de base del 
procedimiento de muestreo. En este sentido, se puso atención en la relevancia de explicitar claramente los conceptos y categorías teóricas usadas para la definición de las elites, así como la descripción detallada de las categorías de clasificación empírica y operacionalización de los indicadores y fuentes de datos.

En segundo lugar, se planteó la posibilidad de utilizar mecanismos y dispositivos de naturaleza intersubjetiva, como la incorporación del juicio de pares expertos para la validación del procedimiento de muestreo y el tamaño de la muestra.

El tercer mecanismo enunciado es el estudio en base a pares expertos con diseño muestral basado en metodologías cuantitativas y cualitativas combinadas.

La introducción de estos mecanismos fortalecen el diseño muestral aplicado a universos de estudio pequeños y de mediano porte -como son las elites y grupos dirigentes- mediante las posibilidades de triangulación de métodos cualitativos y cuantitativos.

\section{BIBLIOGRAFÍA}

Alcántara Manuel, Barragán Mélany, Sánchez Francisco (2018) “Political Career Trajectories and Social Backgrounds Latin American Presidents in Comparative Perspective (1978-2015)" en Alcántara Manuel, Blondel Jean, Thiebault Jean-Louis (2018) Presidents and democracy in Latin American, Routledge, Nueva York.

Bertaux Daniel (2010) L`enquête et ses méthodes. Le récit de vie, (3 edition) Armand Colin, Paris

Becker Howard (2009) Trucos del oficio. Cómo conducir su investigación en ciencias sociales Buenos Aires, Siglo XXI eds.

Bottomore Tom (1967) Elites and Society, NewYork, Routlege.

Cárdenas Julián (2014) El poder económico mundial: análisis de redes de interlocking directorates y variedades de capitalismo, CIS - Centro de Investigaciones Sociológicas, Madrid.

Codato (2016) "Metodologías para a identificaçao das elites. Três exemplos clássicos" en Perissinotto Renato,Codato Adriano(orgs)(2016) Como estudar elites, Curitiba, Ed. UFPR.

Corcuff Philippe (2013) Las nuevas sociologías. Principales corrientes y debates, 1980-2010, Buenos Aires, Siglo XXI eds.

Flick U. (2007 (2004,) Introducción a la investigación cualitativa, Ed.Morata, Madrid, Cap.XVIII y XXI

Genieys William (2011) Sociologie Politique des Élites”, Armand Colin, París.

Connor Anne, Carpenter Barry, Coughlan Barry (2018) "An exploration of key issues in the debate between classic and constructivist grounded theory" en The Grounded Theory Review, Volume 17, issue 1

Creswell John (2009) Research design. Quantitative, qualitative and mixed methods approaches. Sage, Londres.

Dexter, Lewis Anthony. (2006 [1970])Elite and Specialized Interviewing,ECPR Press.

Domhoff G. William (2007) "C. Wright Mills, Floyd Hunter, and 50 years of power structure research" Michigan Sociological Review, Vol. 21 (Fall 2007), pp. 1-54 
Escobar-Perez Jazmine, Cuervo-Martinez Angela (2008) "Validez de contenido y juicio de expertos: una aproximación a su utilización" en Avances en medición, 6, 27-36

Fetters Michael, Molina-Azorin Jose (2017) "The Journal of Mixed Methods Research Starts a New Decade: The Mixed Methods Research Integration Trilogy and Its Dimensions" en Journal of mixed methods, Vol.11(3)

Hennink Monique, Kaiser Bonnie, Weber mary Beth (2019) "What influences saturation? Estimating sample size in focus group research" en Qualitative Health Research, SAGE, 1-14.

Gené Mariana (2014 ) "Sociología política de las elites. Apuntes sobre su abordaje a través de entrevistas" en Revista de Sociología e Política, Vol. 22, n 52, dez. Curitiba.

Glaser Barney.G.y Strauss Anselm L., (2006 [1967]) The Discovery of Grounded Theory Strategies for Qualitative Research, Aldine Transaction, A Division of Transaction Publishers, Rutgers.

Guetterman Timothy (2015) "Descriptions of sampling practices within five approaches to qualitative research in education an the health sciences" en Forum: Qualitative Social Research, Vol.16, n², Art.25, May.2015.

Hartman Michael 2007, The sociology of elites, Routledge, Londres y Nueva York.

Harvey, William S.(2011) "Strategies for Conducting Elite Interviews." Qualitative Research11(4): 431-441.

Hennink Monique, Kaiser Bonnie, Marconi Vincent (2016) "Code saturation versus meaning saturation: how many interviews are enough?" en Qualitative Health Research, SAGE, 1-18.

Hoffmann-Lange Ursula (2018) "Methods of Elite Identification" en Best Heinrich, Higley John(eds) The Palgrave handbook of political elites, Palgrave-Macmillan, Londres.

Keller Franziska Barbara (2018) "Analyses of Elite Networks" en Best Heinrich, Higley John(eds) The Palgrave handbook of polítical elites, Palgrave-Macmillan, Londres.

Mason Mark (2010) "Sample size and saturation in PhD studies using qualitative interviews" en Forum: Qualitative Social Research, Vol11, n³, Art.8, Sept.2010.

Morse Janice (2007) "Sampling in grounded theory" en Bryant Antony, Charmaz Kathy The SAGE Handbook of Grounded Theory, SAGE, Londres.

Ostrander, S.A. (1995) "Surely you're not in this just to be helpful. Access, Rapport, and Interviews in Three Studies of Elites", en Rosanna Hertz, Jonathan Imber (eds)Studyng elites using qualitative method, London, Sage.

Pujadas Muñoz Juan José (1992) El método biográfico: El uso de las historias de vida en Ciencias Sociales, Madrid, Centro de Investigaciones Sociológicas.

Quinn Patton Michael (2002) Qualitative research. Evaluation methods. SAGE, Londres.

Rodríguez-Teruel Juan, Daloz Jean-Pascal (2018) "Surveying and Observing Political Elites" en Best Heinrich, Higley John(eds) (2018) The Palgrave handbook of political elites, Palgrave-Macmillan, Londres.

Ragin Charles (2007 (1994) La construcción de la investigación social. Introducción a los métodos y su investigación, Siglo del Hombre Editores, Universidad de los Andes, Bogotá.

Serna, Miguel (2013) "O viés social na seleção das elites políticas: espaço social e carreira política, um olhar desde o caso uruguaio" en Marenco Andre (org) Os eleitos: Representaçào e carreiras políticas em democracias, Editora UFRGS, Porto Alegre. 
(2004) Revonversao democrática das esquerdas no cone sul, EDUSC-ANPOCS, San Pablo.

Serna, Miguel, Bottinelli Eduardo (2018) "El poder fáctico de las elites empresariales en la política latinoamericana: un estudio comparado de ocho países" en Élites, políticas fiscales y privilegios en América Latina y el Caribe CLACSO - OXFAM, Buenos Aires.

Serna Miguel, Barbero Marcia, González Franco (2015) “Altos ejecutivos de grandes empresas en Uruguay: entre herencia, reconversión y ascenso social" en Marcelo Boado (comp.) El Uruguay desde la sociología 13, Departamento de Sociología, UDELAR, Montevideo.

Serna Miguel (coordinador), Eduardo Bottinelli, Cristian Maneiro, Lucía Pérez, (2012) Giro a la izquierda y nuevas elites en Uruguay: ¿renovación o reconversión?, Publicaciones CSIC-UDELAR, Mdeo.

Stone Lawrence (2011) "Proposografía” en Revista de Sociología e Política,V.19 n³9, jun., pp.115-137.

Thomas R. (1995) "People in big companies" en Rosanna Hertz, Jonathan Imber (eds) Studyng elites using qualitative method, London, Sage.

Ussem Michael (1995) "Reaching corporate executives" en Rosanna Hertz, Jonathan Imber (eds) Studyng elites using qualitative method, London, Sage.

Valles Miguel (2002) Entrevistas cualitativas, Cuadernos Metodológicos 32, Madrid, CIS.

Verd John, López Pedro (2008) "La eficiencia teórica y metodológica de los diseños multimétodo" en Empiria Revista de Metodología de Ciencias Sociales, №16, juldic.

Wainerman Catalina (2001) "Acerca de la formación de investigadores en ciencias sociales" en Wainerman Catalina, Sautu Ruth La trastienda de la investigación, Ed.Lumiere, Buenos Aires. 